\title{
Frequency Discrimination for Amplitude Modulated Sinusoidal Signals at High Carrier Frequencies
}

\author{
A. $\mathrm{SECK}^{a, *}$ AND M. KORDUS ${ }^{b}$ \\ ${ }^{a}$ Institute of Acoustics, Faculty of Physics, Adam Mickiewicz University \\ Umultowska 85, 61-614 Poznań, Poland \\ ${ }^{b}$ Department of Biophysics, Poznań University of Medical Science, Fredry 10, 61-701 Poznań, Poland
}

\begin{abstract}
The current study is a continuation of experiments presented by Sek and Bukała (Acta Physica Polonica A 123 , 1106 (2013). The purpose of the present study was to investigate frequency discrimination of amplitude modulated high frequency carriers. Using $2 \mathrm{AFC}$ procedure, the subjects were presented with two observation intervals of which the first interval contained four pulses of the same high frequency signal (called SSSS), while in the second interval (called SHSH) the second and fourth pulses had higher frequencies values (i.e. shifted upwards by $\Delta f$ ). The carrier frequency (in S pulses) was fixed and equal to $10 \mathrm{kHz}$. Modulation rates were equal to 100, 200, 337, 500, 600, 733, and $800 \mathrm{~Hz}$. The value of the modulation rate was limited to keep all components of the sinusoidal modulation within one auditory filter ( $\sim 17 \%$ of the center frequency) centered at the carrier frequency. Two different types of modulation were used: a simple sinusoidal modulation with the modulation depth $m$ set to $100 \%$, and a logarithmic modulation with the modulation depth $m$ set to $50 \mathrm{~dB}$. Results indicate a strong relationship between frequency discrimination threshold and modulation type. The thresholds are significantly higher for logarithmic modulation in comparison to sinusoidal modulation. Amplitude modulation as well as logarithmic modulation applied to the high frequency carrier cause significant increase in the frequency discrimination threshold. For high frequency sinusoidal signal carriers (i.e. close to $10 \mathrm{kHz}$ ), frequency discrimination thresholds do not depend on amplitude modulation rates up to about $800 \mathrm{~Hz}$. In general, the excitation pattern mechanism was a primary cue enabling frequency discrimination of modulated and unmodulated signals to compare with the mechanism based on the temporal fine structure. However, the excitation pattern was not the only mechanism responsible for the frequency discrimination.
\end{abstract}

DOI: 10.12693/APhysPolA.125.A-149

PACS: $43.66 . \mathrm{Fe}, 43.66 . \mathrm{Hg}$

\section{Introduction}

It has been assumed that the frequency discrimination by normal-hearing subjects is mainly based on temporal information cues derived from phase locking for frequencies up to $4-5 \mathrm{kHz}$ [1-6] whereas above $4-5 \mathrm{kHz}$, discrimination is thought to depend on place mechanisms, based on changes in the excitation pattern $[2,5]$. The supporting arguments come from the behavioral studies [7]. However, some physiological studies by Heinz et al. $[8,9]$, Recio-Spinoso et al. [10] and Temchin et al. [11] suggested the existence of 'residual assignment' of neural impulses to always the same phase of the signal (for frequencies above $5 \mathrm{kHz}$ ). Earlier experiments related to the use of phase locking information by More and Sęk [12] have shown that the majority of subjects was able to discriminate between $\mathrm{H}$ and $\mathrm{I}$ sounds for $F_{0}=800-1000 \mathrm{~Hz}$ with the center frequency of the bandpass filter of $11.1 \mathrm{kHz}$ and $14 \mathrm{kHz}$ (for details, see [12]). It has been shown that the $\mathrm{H}$ and I sound discrimination could not be based neither on the changes in the signal spectrum nor on changes in the excitation pattern. The results indicated that the information about the signal frequency comes from the time distribution of the peaks

*corresponding author; e-mail: oleksek@amu.edu.pl occurring within maxima of amplitude envelope and time distribution of neural pulses. This suggests that the neural impulses synchronization with specific phase of an acoustic wave may reach frequencies about $10 \mathrm{kHz}$ or higher than the previously assumed $5 \mathrm{kHz}[8,12]$. However, this statement is controversial taking into account the well established knowledge on the physiology of hearing. Earlier studies on discrimination of sinusoidal signals by Ritsma et al. [13-15] have shown that the detection threshold deteriorates with an increase in frequency above $5 \mathrm{kHz}$ [14]. However, it is difficult to transfer the conclusion drawn from those experiments onto complex signals. On the other hand, the use of phase locking information for frequencies above $5 \mathrm{kHz}$ is recently an important issue [16] considered also in the context of speech intelligibility for different languages [17-19] or perception of musical intervals. For example, Oxenham et al. [16] have shown that the components of complex tones above $6 \mathrm{kHz}$ produce clearly audible musical pitch and intervals that must involve the phase locking.

In the present study, the frequency discrimination experiment was carried out for high frequency carrier $(10 \mathrm{kHz})$ amplitude modulated at different rates. To be able to see an effect of amplitude envelope superimposed on the carrier, the frequency discrimination thresholds were initially determined for high frequency sinusoidal signal. Then, the same thresholds were determined for 
high frequency carrier being amplitude modulated at different rates. As the phase locking seems to provide more usable information for instantaneous values situated at extreme values of amplitude envelope, two different types of modulation were used, i.e. sinusoidal and logarithmic (see below for exact equations). The most important difference between these types of modulation is that the logarithmic one produces more 'peaky' envelope with a higher crest factor having at the same time more spectral sidebands. It was expected that the amplitude modulation might lead to an improvement of the frequency discrimination, especially for the amplitude modulator characterized by a higher crest factor.

\section{Aim of the study}

The frequency discrimination of the $\mathrm{H}$ and I sounds presented by Moore and Sęk [12] and Sęk and Bukała [20] suggested an effective use of a residual phase locking connected with instantaneous extreme values. On the other hand, the results suggested an effective use of the temporal fine structure included in the signals, especially when extreme values coincided with maxima in amplitude envelope. Therefore, the question whether introducing a sinusoidal envelope on a high frequency carrier may influence the frequency discrimination of the carrier seems to be quite crucial. To answer this question was the main purpose of the presented paper.

\section{Materials and method}

\subsection{Method}

The study was based on a modified computer program and method presented by Sęk and Moore [21]. The modification allowed to generate the amplitude modulated high frequency carrier using different modulators. In accordance with the $2 \mathrm{AFC}$ procedure, the subjects were presented with two observation intervals in a random order. One interval consisted of four identical high frequency pulses (marked as SSSS) while the other observation interval (marked as SHSH), consisted of four pulses of different frequency. However, the second and forth pulse in that interval had higher frequencies (i.e. shifted upwards by $\Delta f$ ). When amplitude modulation was used, the identical modulation (i.e. depth and rate) were used with respect to all pulses in the first and in the second interval. Each pulse in the SSSS and SHSH tones lasted for $200 \mathrm{~ms}$ including $20 \mathrm{~ms}$ of rise and fall time (cosine square function) and was separated from the adjacent sound ( $\mathrm{S}$ or $\mathrm{H}$ ) with a $100 \mathrm{~ms}$ interval of silence. The time between observation intervals was $300 \mathrm{~ms}$. The overall level of signals was equal to $50 \mathrm{~dB}$ SL and was adjusted based on absolute threshold measured prior to the experimental session. The carrier frequency (in $\mathrm{S}$ pulses) was fixed and equal to $10 \mathrm{kHz}$. Two different types of modulation were used. The first one was a simple sinusoidal modulation with the modulation depth $m$ set to $100 \%$ that can be described by means of the following equation:

$$
a(t)=\left(1+m \sin \left(2 \pi f_{m} t\right)\right)\left(\sin 2 \pi f_{c} t\right),
$$

where $f_{m}$ and $f_{c}$ are the modulation rate and the carrier frequency, respectively. The logarithmic modulation mentioned above can be described by the following formula:

$$
a(t)=10^{\frac{r \sin \left(2 \pi f_{m} t+\theta\right)}{40}} \sin \left(2 \pi f_{c} t\right),
$$

where $r$ denotes modulation depth that was set to $50 \mathrm{~dB}$.

Examples of the above signals are depicted in Fig. 1. The top panel presents a portion of a waveform obtained by means of sinusoidal amplitude modulation of a $10-\mathrm{kHz}$ carrier at a rate of $500 \mathrm{~Hz}$, while the lower one shows logarithmic modulation for modulation depth set to $50 \mathrm{~dB}$. As can be seen from this graph, the logarithmic modulation results in more 'peaky' waveform and contains smaller number of extreme instantaneous values within maxima of amplitude envelope. Therefore one may expect that this waveform may produce very prominent time intervals between neural spikes correlated with extreme instantaneous values of the signal. Logarithmic modulation results also in much higher crest factor $(\mathrm{CF})$ which is also specified in the respective panels.

Modulation rates used in the experiment were 100, 200, $337,500,600,733$, and $800 \mathrm{~Hz}$. The value of the modulation rate was limited to keep all components of the sinusoidal modulation within one auditory filter $(\sim 17 \%$ of the center frequency) centered at the carrier frequency. This limitation enabled a direct comparison of the results acquired for unmodulated and (sinusoidally) modulated signals, as the spectra of both types of signal were falling within one auditory filter. The spectrum of the logarithmic modulation that contains more than two sidebands (especially for chosen modulation depth) and the results acquired using this type of modulation will be considered separately.

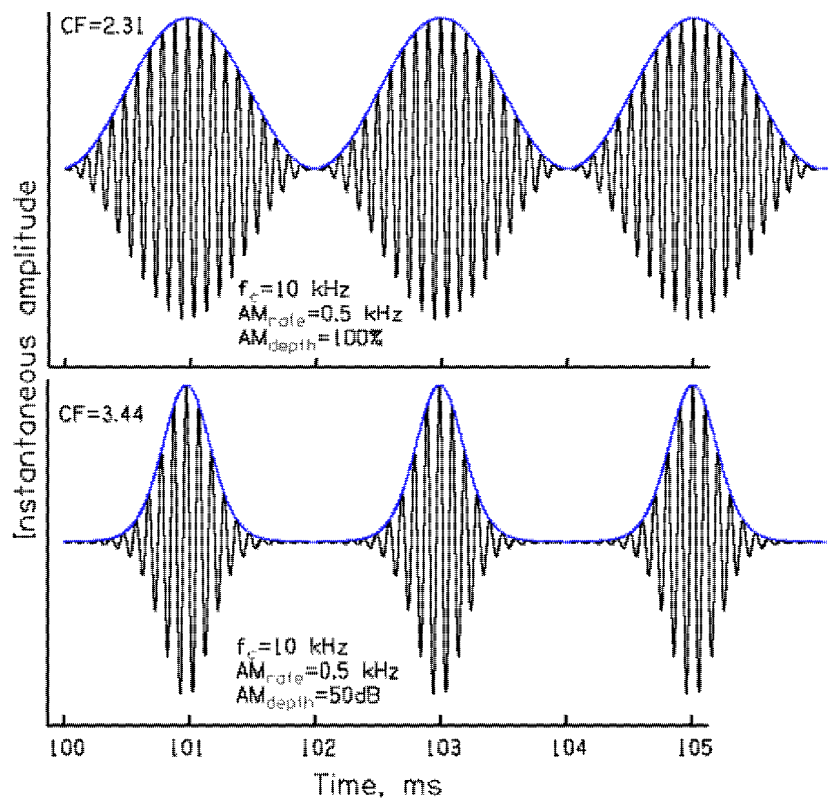

Fig. 1. The top panel presents example of a waveform of a $10-\mathrm{kHz}$ carrier with sinusoidal amplitude modulation at a rate of $500 \mathrm{~Hz}$, while the lower one shows logarithmic modulation for modulation depth set to $50 \mathrm{~dB}$ (see detailed equations in text). 
In the experiment, the task of the subject was to identify the interval which included changes concerning the presentation of the SHSH series. The experiment began with a high, clearly audible value of $\Delta f$, so as to allow the subjects to discriminate the stimuli presented in the initial stage of each experimental run. Two successive correct responses brought about a reduction of the frequency difference, $\Delta f$, and one incorrect answer brought about an increase of the difference. Eight turnpoints were determined in a single experimental run. The change in the frequency difference, $\Delta f$, was multiplied (or divided) by $1.25^{3}$ until the first turnpoint, then by $1.25^{2}$ up to the second turnpoint, and by $1.25^{1}$ for the remaining six turnpoints on which the threshold was calculated. The threshold values were obtained on the basis of five independent measurements.

\subsection{Subjects}

Seven subjects (age up to 27) took part in this experiment. All of them were paid for their services. They had normal hearing in the range up to $14 \mathrm{kHz}$ which was analyzed by using Grason-Stadler GSI61 audiometer. All subjects were given a series of training sessions for about 4-6 hours. This was done to familiarize the subjects with their task and to make the test procedure more clear to them.

\subsection{Apparatus}

The signals were generated using the Sound Blaster Audigy audio card with a 24 bit resolution at $48 \mathrm{kHz}$ sampling rate controlled by a PC with installed software proposed by Sęk and Moore [21]. The signals were presented monaurally via ER-2A insert earphones to the ear chosen by the subject. During the study, the subjects were in a soundproof booth with the computer peripheries necessary for answering questions concerned with testing.

\section{Results}

Results of the frequency discrimination test are presented in Fig. 2. Thresholds are plotted as functions of amplitude modulation rate. Each panel presents the data for one subject. The lower right panel depicts thresholds averaged over all subjects. Open squares in each panel represent the frequency discrimination thresholds, $\Delta f$, for signals being amplitude modulated by means of a sinusoidal signal (linear modulation), while open circles represent the thresholds for logarithmic modulation. Vertical lines denote \pm one standard error calculated based on five repetitions of measurement for each set of parameters (i.e. modulation rate and modulation type). Some of the datapoints were shifted in frequency domain to avoid overlapping. The frequency discrimination thresholds acquired for high frequency unmodulated sinusoid are presented by means of shaded horizontal bars in each panel. Heights of the bars represent areas of \pm one standard error of actual values of the thresholds whose exact values are situated (but not plotted) in the middle (along) each bar.

The frequency discrimination threshold for all types of signals is characterized by a variability across the subjects. For the linear and the logarithmic modulation type, three patterns of result accompanied by differences in subjects' performance were observed. In general, the data acquired for subjects S1 and S2 are characterized by a low thresholds $\Delta f$ ranging from $20 \mathrm{~Hz}$ to $40 \mathrm{~Hz}$, while for subjects S3 and S4 the thresholds are within the range $80-100 \mathrm{~Hz}$. The highest discrimination thresholds, ranging from $90 \mathrm{~Hz}$ to $130 \mathrm{~Hz}$, were observed for subjects S5, S6, and S7. Based on the presented raw data it is very difficult to draw a consistent conclusion about dependence of the discrimination threshold on the modulation rate. The results averaged over all subjects suggest, however, that the modulation rate did not influence markedly the threshold. Moreover, it seems clear that the difference between modulation types may be significant. It is worth to add that, on average, the obtained results are broadly consistent with the data collected and published by Wier et al. [22], as well with the data by presented by Sęk and Moore [5].

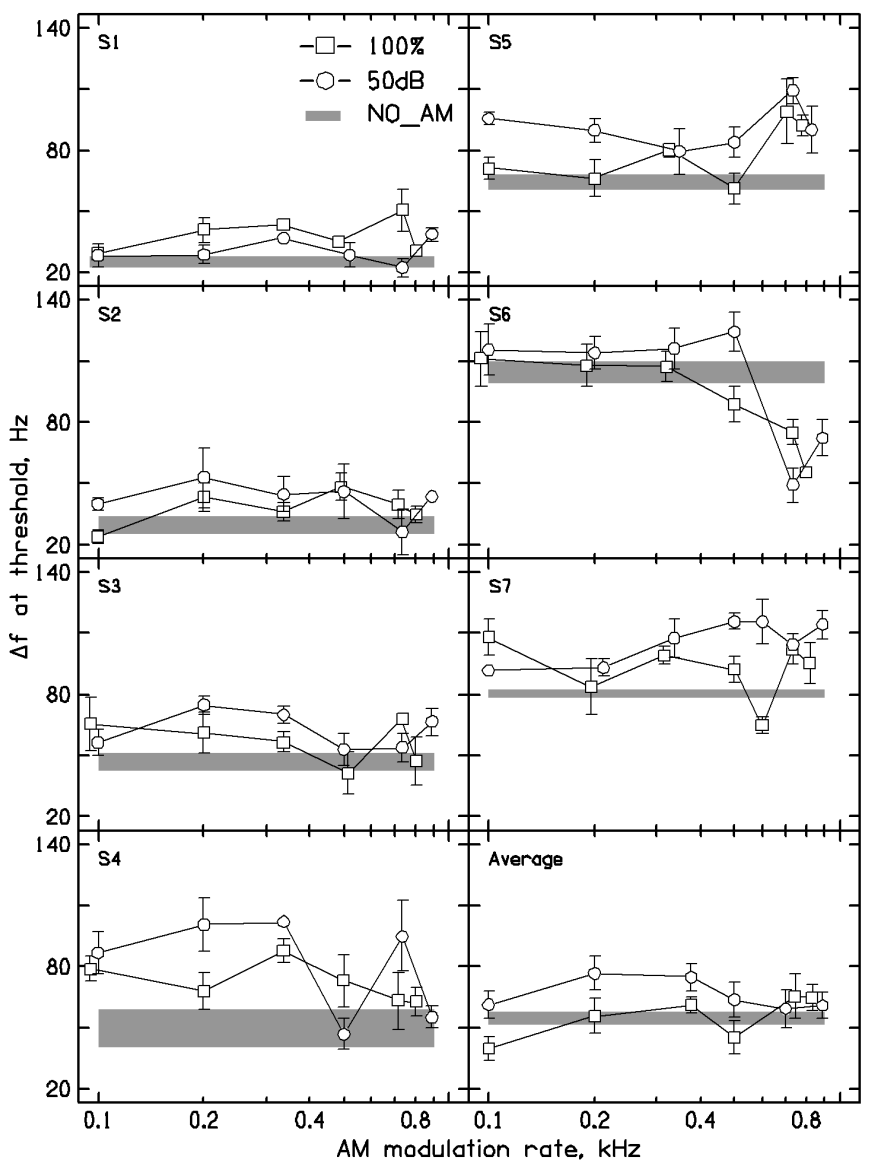

Fig. 2. The frequency discrimination thresholds, $\Delta f$, for unmodulated and amplitude modulated sinusoidal signals at high frequencies as functions of the modulation rate. Open squares represent $\Delta f$ for linear modulation, open circles for logarithmic one. Vertical lines denote \pm one standard error. The frequency discrimination thresholds for unmodulated signal are presented by means of shaded horizontal bars. The height of the bars represents areas of \pm one standard error of actual value of the thresholds. 
Raw results obtained for modulated signals, i.e. just noticeable frequency difference, were subjected to an analysis of variance (ANOVA) using GENSTAT statistical package [23]. A within-subject two-way analysis of variance was carried out with the factors of modulation type (including $\mathrm{NO}_{-} \mathrm{AM}$ case) and modulation rate. The within-subject $\bar{A}$ VOVA treats the data collected for each subject as a repetition of a single measurement and allows to draw more general conclusion about the analyzed phenomenon. It turned out that modulation rate was not statistically significant $[F(5,30)=0.953$, $p=0.462]$. However, the modulation type was statistically significant $[F(2,12)=8.666, p=0.0005]$. The interaction of the modulation rate and the modulation type was not statistically significant $[F(10,60)=0.96$, $p=0.487]$ suggesting that the differences between the discrimination thresholds for modulated sounds did not change with the modulation rate.

The post-hoc Tuckey test revealed that the data obtained for all modulation rates created a single homogenous group and there were no statistically significant differences between averaged data for any two modulation rates. This proves that, on average, the discrimination thresholds did not depend on the modulation rate. However, the same test applied to different modulation types (including $\mathrm{NO}_{-} \mathrm{AM}$ case) revealed statistically significant differences between each of two (out of three) groups of the data, i.e. (AM $100 \%$ vs. AM $50 \_\mathrm{dB}, p=0.003$; AM $100 \%$ vs. NO ${ }^{-} \mathrm{AM}, p<0.0 \overline{01}$; $\overline{\mathrm{AM}} 50 \mathrm{~dB}$ vs. $\left.\mathrm{NO}_{-} \mathrm{AM}, p<0.001\right)$. The lowest frequency discrimination thresholds were obtained for unmodulated sounds while the highest ones for the logarithmic modulation.

Based on the above presented analysis it can be stated that the frequency discrimination thresholds for high frequency carrier being amplitude modulated do not depend on the modulation rate but they do depend on the modulation type, including the NO_AM case. This sort of result is somehow surprising as initially it was suggested that the temporal fine structure combined with the phase locking would provide some additional cues in frequency discrimination. This should make the subjects' performance slightly better, especially in case of extremes of instantaneous values coinciding with maxima in amplitude envelope, what actually happened while presenting modulated signals. However, such improvement was not observed: the frequency discrimination thresholds were higher when modulation was introduced on a high frequency carrier.

Therefore, searching for an explanation of this experimental finding, the excitation pattern model [24] was explored and used. If the differences in the excitation patterns evoked by $\mathrm{H}$ and $\mathrm{S}$ (calculated separately for each type of modulation) signals would correspond with the measured averaged thresholds, then the excitation pattern mechanisms were the primary cue in frequency discrimination of high frequency sounds.

Using the model described by Moore et al. [25], the differences in excitation patterns evoked by the $\mathrm{S}$ and $\mathrm{H}$ signals (for the averaged discrimination thresholds $\Delta f$ ), were calculated. Standard MATLAB function ( $p s d[26]$ ) was used to analyze spectral structure of signals with a resolution of $2 \mathrm{~Hz}$. The spectra were treated as an input for the calculation of excitation patterns [25]. The statistical analysis of the acquired data showed that the discrimination thresholds were, on average, independent of modulation rate being at the same time significantly higher for modulated sounds.

Detection/discrimination cue based on the excitation pattern differences was initially suggested by Zwicker and Fastl [27]. They argued that if the difference of excitation patterns caused by two signals in any frequency area is greater than $1 \mathrm{~dB}$, than the signals should be assessed as different. Therefore, the calculation of excitation patterns evoked by $\mathrm{S}$ and $\mathrm{H}$ signals was done, taking into account the averaged discrimination threshold, $\Delta f$, both for the unmodulated signal and separately for each type of modulated sounds.

As the results of differences in excitation patterns across all modulation rates were very similar, an example result, for modulation rate of $733 \mathrm{~Hz}$, in the frequency range above $7 \mathrm{kHz}$, is shown in Fig. 3 .

The top line of the figure shows excitation pattern for $\mathrm{S}$ (solid line) and $\mathrm{H}$ (dashed line) signals, respectively, while the bottom row presents the differences between these excitations. Subsequent columns of the figure illustrate the data for (unmodulated) sinusoidal signal and two different types of modulation. As it can be seen from the bottom panels of Fig. 3, for all signal types the difference in excitation patterns reaches values higher than $1 \mathrm{~dB}$.
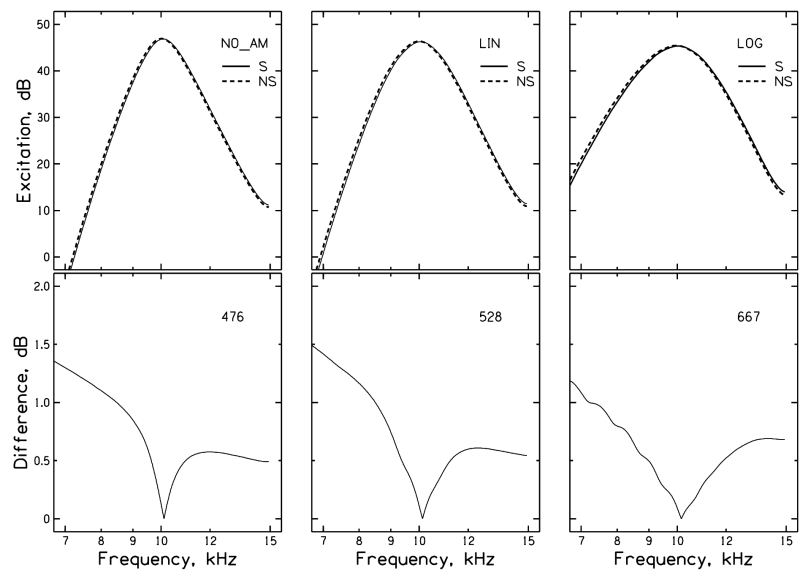

Fig. 3. Excitation patterns for $\mathrm{S}$ (solid line) and $\mathrm{H}$ (dashed line) signals (upper row) and differences in respective excitation patterns (lower row). Subsequent columns illustrate data for (unmodulated) sinusoidal signal and two different modulation types (i.e. linear and logarithmic modulation, respectively).

The model described by Moore et al. [25] took into account properties of the basilar membrane (i.e. its tonotopic organization) and provided excitation patterns 
(in $\mathrm{dB}$ ) on a logarithmic frequency scale at its output. This way it is possible to calculate overall excitation pattern differences (in certain frequency band) in some arbitrary units.

The overall excitation pattern differences, i.e. the differences summed up in all analyzed frequency bands, are listed as numbers (in arbitrary units) in each panel of the bottom row. They reach the highest values for logarithmic modulation being at the same time the lowest for unmodulated sounds. The order of the overall excitation pattern differences reflects the obtained averaged results. This suggests that the excitation pattern differences could be a primary cue in the frequency discrimination. However, if the excitation pattern differences were the only detection cue in the frequency discrimination task, the summed up differences should reach much closer values. Marked differences between them suggest that apart from the excitation pattern cues, some other mechanism(s) must have been involved in the frequency discrimination task.

\section{Discussion}

Results presented by Moore and Sęk [12] and Sęk and Bukała [20] suggested that subjects could use residual phase locking connected with instantaneous extreme values of sound pressure situated in the maxima of amplitude envelope. This conclusion was drawn based on the analysis of the output of a single auditory filter centered at a high frequency. The output signal had a very prominent amplitude envelope that was similar to amplitude modulation. Moreover, the above-quoted authors stated that the excitation pattern differences produced by $\mathrm{H}$ and I sounds did not play any role in the discrimination. Therefore, the question whether introducing a sinusoidal envelope on a high frequency carrier may influence the frequency discrimination threshold of the carrier seemed to be quite crucial and was the main purpose of the present paper. It is well known that a single auditory neuron is capable to generate no more than several hundred pulses per second [28]. Each pulse is caused by a temporal depolarization of the inner hair cell [29]. The probability of occurrence of an impulse is monotonically dependent on the amplitude of the stimulating waveform [30]. Therefore, generation of an impulse seems to be more probable for extreme instantaneous values in the maximum amplitude envelope. Impulses do not appear in the adjacent extreme values of the envelope when its repetition rate is higher than a few hundred times per second. However, each impulse coincides with a local maximum of an envelope. Innervation of the basilar membrane is very dense, and then there are at least several afferent neurons that innervate each of the inner hair cells. One may expect therefore that each maximum of amplitude envelope will have its unique representation in the series of pulses observed in a group of neurons. Time distances between adjacent spikes being close to successive maxima of envelope but assigned directly to the extreme values of instantaneous values provide precise information about frequency of the signal. In case on an unmodulated sinusoidal signal, situation is quite different. The amplitude envelope is constant and therefore neural spikes may be expected at any time when an instantaneous value maximum occurs. There will be no prominent (i.e. the most frequently repeated) time interval that could be used to assess the frequency of the signal. The distribution of the intervals will be approximately uniform within certain range. Therefore the frequency discrimination of a high frequency unmodulated sinusoid should be worse.

Results of the present experiment have shown that imposing amplitude modulation on a high frequency sinusoidal waveform did not improve frequency discrimination. Rather an opposite effect was observed: the lowest thresholds were observed for unmodulated sinusoidal signal. Moreover, the thresholds for logarithmic modulation, the one that produces very prominent peaky amplitude envelope, reached the highest values. This means that temporal fine structure and the neural pulses associated with the maxima in instantaneous values, located in the extreme values of the amplitude envelope, even if they would provide additional information related to the frequency of the signal, did not make the frequency discrimination task easier. Similar situation was also observed for sinusoidal amplitude modulation, i.e. the modulation that produces less peaky envelope, with more extreme instantaneous values within maxima in the amplitude envelope. The frequency discrimination thresholds for this type of amplitude modulation were significantly lower than those obtained for logarithmic modulation but on the other hand, significantly higher than those observed for unmodulated sounds. In general, the phase locking was not a primary cue that enabled the frequency discrimination in the experiment presented in this paper.

The other possible mechanism that could provide effective information enabling the frequency discrimination is the excitation pattern mechanism. The analysis of differences in the excitation patterns for signals $\mathrm{S}$ and $\mathrm{H}$, for frequency shift corresponding to average discrimination threshold showed similar results. For all modulation rates the result was similar, i.e. the highest overall difference in excitation patterns summed up in frequency bands above $7 \mathrm{kHz}$ were obtained for the logarithmic modulation, while the lowest one for the unmodulated sounds. Therefore one can say that a certain correlation between the excitation pattern differences and the frequency discrimination thresholds was observed. However, it is difficult to state that the excitation pattern was the only or a primary mechanism that enabled the frequency discrimination. If the excitation pattern mechanism were the only mechanism responsible for the discrimination, then the differences in excitation pattern at threshold should be very similar but actually, they were not. One possible reason for which the excitation pattern mechanism did not provide sufficient explanation of the observed effect is the lack of knowledge about frequency resolution of the auditory system in the high fre- 
quency area, especially about the auditory filter shape (symmetry) and bandwidth. Direct measurements of the psychophysical tuning curves (PTCs) $[31,32]$ characterizing the auditory filter shape by means of the notched noise method [33] would provide more detailed information concerning this issue. In summary, the presented results indicate that the frequency discrimination of high frequency sounds is not mediated by a single mechanism, such as the excitation pattern or phase locking, alone.

\section{Conclusions}

The results of the experiment presented in this paper allow to formulae the following conclusions:

1. Frequency discrimination thresholds for high frequency (i.e. close to $10 \mathrm{kHz}$ ) sinusoidal signal are approximately independent of amplitude modulation rate up to $800 \mathrm{~Hz}$.

2. Amplitude modulation (both sinusoidal at $100 \%$ and logarithmic at $50 \mathrm{~dB}$ ) applied to the high frequency carrier brings about a significant increase in the frequency discrimination threshold.

3 . The frequency discrimination threshold strongly depends on modulation type. The threshold is significantly higher for logarithmic modulation compared to sinusoidal one.

4. Results do not indicate that the temporal fine structure plays a significant role in frequency discrimination of amplitude modulated carrier at frequencies close to 10 $\mathrm{kHz}$.

5. The excitation pattern was not the only mechanism that enabled the frequency discrimination of modulated and unmodulated signals. However, a certain correlation between the excitation pattern differences and the frequency discrimination thresholds for each signal type was observed.

\section{Acknowledgments}

This work was supported by a grant from the from the National Science Centre No. N N 518502139.

\section{References}

[1] B.C.J. Moore, J. Acoust. Soc. Am. 54, 888 (1973).

[2] B.C.J. Moore, J. Acoust. Soc. Am. 54, 610 (1973).

[3] B.C.J. Moore, J. Acoust. Soc. Am. 55, 359 (1974).

[4] J.L. Goldstein, P. Srulovicz, in Psychophysics, Physiology of Hearing, Eds. E.F. Evans, J.P. Wilson, Academic Press, London 1977, p. 337.

[5] A. Sek, B.C. Moore, J. Acoust. Soc. Am. 97, 2479 (1995).

[6] C. Micheyl, R.P. Carlyon, J. Acoust. Soc. Am. 104. 3006 (1998)

[7] B.C.J. Moore, Introduction to Hearing Psychology, I st. ed., PWN, Poznań 1999.
[8] M.G. Heinz, H.S. Colburn, L.H. Carney, Neur. Comp. 13, 2317 (2001).

[9] M.G. Heinz, H.S. Colburn, L.H. Carney, J. Acoust. Soc. Am. 110, 2065 (2001).

[10] A. Recio-Spinoso, A.N. Temchin, P. van Dijk, Y.H. Fan, M.A. Ruggero, J. Neurophys. 93, 3615 (2005).

[11] A.N. Temchin, A. Recio-Spinoso, P. van Dijk, M.A. Ruggero, J. Neurophys. 93, 3635 (2005).

[12] B.C.J. Moore, A. Sęk, J. Acoust. Soc. Am. 125, 3186 (2009).

[13] R.J. Ritsma, Int. Audiol. 2, 34 (1963).

[14] R.J. Ritsma, J. Acoust. Soc. Am. 34, 1224 (1962).

[15] R.J. Ritsma, J. Acoust. Soc. Am. 35, 1241 (1963).

[16] A.J. Oxenham, C. Micheyl, M.V. Keebler, J. Acoust. Soc. Am. 125, 2189 (2009).

[17] K. Hopkins, B.C.J. Moore, J. Acoust. Soc. Am. 125 442 (2009).

[18] A. Wicher, E. Ozimek, E. Szymiec, Acta Phys. Pol. A 118, 179 (2010).

[19] K. Hopkins, B.C.J. Moore, J. Acoust. Soc. Am. 127, 1595 (2010)

[20] A. Sęk, M. Bukała, Acta Phys. Pol. A 123, 1106 (2013).

[21] A. Sęk, B.C.J. Moore, Int. J. Audiology 53, 58 (2012).

[22] C.C. Wier, W. Jesteadt, D.M. Green, J. Acoust. Soc. Am. 61, 178 (1977)

[23] P. Lane, N. Galwey, N. Alvey, Genstat 5. An Introduction, Clarendon Press, Oxford 1987.

[24] B.R. Glasberg, B.C.J. Moore, Hear. Res. 47, 103 (1990).

[25] B.C.J. Moore, B.R. Glasberg, T. Baer, J. Audio Eng. Soc. 45, 224 (1997).

[26] Mathworks, Matlab \%. Signal precessing toolbox, Mathworks Inc. 2005.

[27] E. Zwicker, H. Fastl, Psychoacoustics - Facts, Models, Second Edition, Springer-Verlag, Berlin 1999.

[28] J.O. Pickles, An Introduction to the Physiology of Hearing, 2nd ed, Academic Press. London 1988.

[29] J.F. Ashmore, J. Physiol. 338, 323 (1987).

[30] A.R. Palmer, in: Hearing, Ed. B.C.J. Moore, Academic Press, San Diego 1995, p. 75.

[31] A. Sęk, J. Alcántara, .B.C.J. Moore, K. Kluk, A. Wicher, Int. J. Audiology 44, 408 (2005).

[32] A. Sęk, A. Wicher, S. Drgas, Archiv Acoust, 32, 707 (2007).

[33] R.D. Patterson, J. Acoust. Soc. Am. 59, 640 (1976). 